\title{
Complex line bundles in relativity
}

\author{
W. D. Curtis \\ Department of Mathematics, Kansas State University, Manhattan, Kansas 66502 \\ D. E. Lerner
}

Department of Mathematics, University of Kansas, Lawrence, Kansas 66045

(Received 17 August 1977)

We exhibit the complexified spin and conformally weighted functions as sections of holomorphic line bundles over $P_{1}(C) \times P_{1}(C)$. As an example of a nontrivial bundle, we discuss the complex null cone in some detail.

\section{INTRODUCTION}

In recent work on general relativity, certain weighted scalars known as "spin and conformally weighted functions" have played a prominent role, During the past few years, in conjunction with work on complex spacetime and twistor theory, ${ }^{1,2}$ it has become necessary to consider the "complexifications" of these functions. Our purpose in this paper is to identify these scalars as sections of certain line bundles and to discuss some of their properties from this point of view.

Since the material related to the real two-sphere is already known in another form, ${ }^{3}$ we shall just review it briefly and concentrate our attention on the holomorphic line bundles which appear in the discussion of complex space-times. Most of the formalism for the real case, developed in Sec. 2, carries over virtually unchanged, although some subtle and important differences do occur. For example, in Sec. 3, we show that the complex null cone is actually a nontrivial $C^{*}$-bundle over its space of generators. In Sec. 4 , we exhibit complex null infinity as a nontrivial bundle and examine its holomorphic global cross sections; these are the "good cuts" of Newman and his coworkers." 1,4 Each cut is doubly ruled by the asymptotic twistors of Penrose. ${ }^{2}$ Those asymptotic twistors "not entirely on $C$ " are shown to be line bundles obtained from complex null infinity by suitable restrictions.

\section{PRELIMINARIES}

We begin by reviewing two ways of constructing line bundles (i.e., one complex-dimensional vector bundles) over a differentiable manifold $M_{\bullet}{ }^{5,6}$

The first method involves patching together the trivial bundles $\left\{U_{\alpha} \times C\right\}$ over an open cover $\left\{U_{\alpha}\right\}$ of $M$. Suppose given, for each nonempty double intersection, a map $h_{\alpha \beta}: U_{\alpha} \cap U_{\beta} \rightarrow C^{*}$, where $C^{*}$ is the multiplicative group of nonzero complex numbers. Provided that $h_{\alpha \beta} h_{\beta \gamma}=h_{\alpha \gamma}$ in any nonempty triple intersection, we can glue these bundles together in a consistent fashion: If $x \in U_{\alpha} \cap U_{\beta}$, then the pair $\left(x, z_{\beta}\right)$ in $U_{\beta} \times C$ is identified with $\left(x, h_{\alpha \beta}(x) z_{\beta}\right)$ in $U_{\alpha} \times C$. The transition functions $\left\{h_{\alpha \beta}\right\}$ determine the line bundle completely. A section $s$ of the bundle is given by a set of maps $\left\{s_{\alpha}: U_{\alpha} \rightarrow C\right\}$ satisfying $s_{\alpha}=h_{\alpha \beta} s_{\beta}$ in $U_{\alpha} \cap U_{\beta} ; s_{\alpha}$ is called the local representative of $s$ in $U_{\alpha}$. The bundle is $C^{\infty}$ provided that each $h_{\alpha \beta}$ is $C^{\infty}$; if $M$ is a complex manifold and each $h_{\alpha \beta}$ is holomorphic, the line bundle is said to be holomorphic.
The second construction begins with a principal bundle $\pi: P \rightarrow M$ with structure group $G .{ }^{5}$ Let $\rho$ be a representation of $G$ on $C$. Define an equivalence relation on $P \times C$ by $(p, z) \sim\left(p g, \rho\left(g^{-1}\right) z\right)$ for all $g \in G$, and denote the equivalence class of $(p, z)$ by $\{p, z\}_{0}{ }^{7}$ The set of all equivalence classes, $B(\rho)$, is a line bundle over $M$ with projection $\pi(\{p, z\})=\pi(p)$. In terms of the previous construction, it is not difficult to verify that if $P$ is defined by the transition functions $\left\{\gamma_{\alpha \beta}\right\}$, then $B(\rho)$ is defined by the transition functions

$$
h_{\alpha \beta}=\rho\left(r_{\alpha \beta}\right) \text {. }
$$

Now suppose we are given a section $s: M \rightarrow B(\rho)$. Let $p \in P$ with $\pi(p)=x$. Denote the component of $s(x)$ in the frame $p$ by $\widetilde{s}(p)$ :

$$
s(\pi(p))=\{p, \widetilde{s}(p)\} \text {. }
$$

This defines a complex-valued function on the principal bundle $P$. Note that, for $g \in G, s(\pi(p))=\{p, \tilde{s}(p)\}$ $=\{p g, \tilde{s}(p g)\}$, so that

$$
\tilde{s}(p g)=\rho\left(g^{-1}\right) \tilde{s}(p) \text {. }
$$

Conversely, any function satisfying (1.3) gives rise to a section of $B(\rho)$; this correspondence is $1-1$.

To relate this alternative construction to the first one, let $\left\{U_{\alpha}\right\}$ be an open cover of $M$ such that for each $\alpha$ there exists a local cross section $e_{\alpha}: U_{\alpha} \rightarrow P \mid U_{\alpha}$. Then each $\{p, z\} \in B(\rho) \mid U_{\alpha}$ has a unique representative $\left(e_{\alpha}, z_{\alpha}\right)$, and we assign to $\{p, z\}$ the local coordinates $\left(\pi\left(e_{\alpha}\right), z_{\alpha}\right) \in U_{\alpha} \times C$. In $U_{\alpha} \cap U_{\beta}$, we have $e_{\alpha}=e_{\beta} r_{\beta \alpha}$, where $r_{\beta \alpha}: U_{\beta} \cap U_{\alpha} \rightarrow G$, and the transition functions for $B(\rho)$ are given by (1.1). If $s$ is a section of $B(\rho)$, its local representative in $U_{\alpha}$ is given simply by

$$
s_{\alpha}=\tilde{s}\left(e_{\alpha}\right) \text {. }
$$

We shall use this construction extensively in what follows.

\section{SPIN AND CONFORMALLY WIEGHTED FUNCTIONS ON $S^{2}$}

Consider the principal $C^{*}$-bundle $\pi: C^{2}-\{0\}$ $\rightarrow P_{1}(C) \cong S^{2}$ determined by $\pi:\left(\xi^{0}, \xi^{1}\right) \rightarrow\left[\xi^{0}, \xi^{1}\right]$ (homogeneous coordinates). For any complex number $w$ and any integer or half-integer $s$, the mapping $(s, w): \lambda$ $\rightarrow \lambda^{(s-w)_{\lambda}^{-}-(s+w)}$ is a representation of $C^{*}$ on $C$; using this, we construct a line bundle $B(s, w) \rightarrow s^{2}$. By means 
of (1.3), a cross section of $B(s, w)$ may be identified with a function $f\left(\xi^{0}, \xi^{1}, \bar{\xi}^{0^{\prime}}, \bar{\xi}^{1^{*}}\right)=f\left(\xi^{A}, \bar{\xi}^{A^{\prime}}\right)$ homogeneous of degree $(w-s, w+s)$ :

$$
f\left(\lambda \xi^{A}, \bar{\lambda} \bar{\xi}^{A^{\prime}}\right)=\lambda^{w-s} \bar{\lambda}^{w+s} f\left(\xi^{A}, \bar{\xi} A^{\prime}\right), \quad \lambda \in C^{*} .
$$

\{Technically [cf. (1.3)], we should write the argument of $f$ as $\xi^{A} \lambda$; obviously, $\left.\xi^{A} \lambda=\lambda \xi^{A}.\right\}$

The sections of $B(s, w)$ are called functions of spin weight $s$ and conformal weight $w$. To see that this agrees with the usual definition, it is necessary to introduce a particular local trivialization of the principal bundle:

For each $\beta=\left(\beta_{B}^{A}\right) \in \mathrm{SL}(2, C)$, define a local complex coordinate on $S^{2}$ by $\xi_{\beta}\left(\left[\xi^{0}, \xi^{1}\right]\right)=\xi_{B}^{0} / \xi_{B}^{1}$, where $\xi_{\beta}^{A}=\beta_{B}^{A} \xi^{B}$. The domain of $\underline{\zeta}_{\beta}$ is the open set $U_{B}$ defined by $\xi_{B}^{1} \neq 0$. Put $P_{\beta}=\left(1+\zeta_{\beta} \bar{\zeta}_{\beta}\right)^{1 / 2}$ and define a local cross section $e_{\beta}: U_{\beta} \rightarrow C^{2}-\{0\} \mid U_{\beta}$ by

$$
e_{\beta}=\left(\xi^{0} / \xi_{\beta}^{1} P_{\beta}, \xi^{1} / \xi_{\beta}^{1} P_{\beta}\right) \text {. }
$$

If $\alpha$ is another element of SL $(2, \mathrm{C})$, then clearly

$$
e_{\alpha}=e_{\beta}\left(\xi_{\beta}^{1} P_{\beta} / \xi_{\alpha}^{1} P_{\alpha}\right) \text { in } U_{\alpha} \cap U_{\beta} \text {. }
$$

Thus $\gamma_{\alpha \beta}=\xi_{\alpha}^{1} P_{\alpha} / \xi_{\beta}^{1} P_{\beta}$; and using (1. 1), the transition functions for $B(s, w)$ are given by

$$
\begin{aligned}
h_{\alpha \beta} & =\left(r_{\alpha \beta}\right)^{s-w}\left(\overline{r_{\alpha \beta}}\right)^{-s-w} \\
& =\left(\frac{\xi_{\alpha}^{1} / \xi_{\beta}^{1}}{\bar{\xi}_{\alpha}^{1}}\right)^{s}\left(\frac{1+\xi_{\beta}^{1}}{\left|\xi_{\alpha}^{0} / \bar{\xi}_{\beta}^{1}\right|^{2}+\left|\xi_{\alpha}^{1} / \xi_{\beta}^{1}\right|^{2}}\right)^{w} .
\end{aligned}
$$

If we let $\left(\begin{array}{cc}a & b \\ c & d\end{array}\right)=\alpha \beta^{-1}$, and note that $\zeta_{\alpha}=\left(a \zeta_{\beta}+b\right) /\left(c \zeta_{\beta}+d\right)$, this becomes

$$
h_{\alpha \beta}\left(\zeta_{\beta}, \bar{\zeta}_{\beta}\right)=\left(\frac{c \zeta_{\beta}+d}{\bar{c} \bar{\zeta}_{\beta}+\bar{d}}\right)^{s}\left(\frac{1+\zeta_{\beta} \bar{\zeta}_{\beta}}{\left|a \zeta_{\beta}+b\right|^{2}+\left|c \zeta_{\beta}+d\right|^{2}}\right)^{w} .
$$

Recalling that the local representatives $\left\{s_{\alpha}\right\}$ of a section of $B(s, w)$ satisfy $s_{\alpha}=h_{\alpha \beta} s_{\beta}$, we have shown that these are precisely the functions of spin weight $s$ and conformal weight $w$ as defined in Refs. 3,8 .

It should be noted that although these bundles are all different from the standpoint of representation theory, ${ }^{9}$ they are not all topologically distinct. In fact $B(s, w)$ is isomorphic to $B(s, 0)$, for all $w$ 。 One way to see this is to observe that the defining representations $(s, w)$ and $(s, 0)$ are homotopic to one another (Ref。 5 , pp. pp. $27-29)$, the homotopy being given simply by $\{(s, t w): t \in[0,1]\}$ 。 To conclude this section, we recall that any smooth line bundle is completely characterized by its Chern class, ${ }^{5}$ which for $S^{2}$ is an element of $H^{2}\left(S^{2} ; Z\right) \cong Z$. Though we do not give the proof here, it is not difficult to show that the Chern class of $B(s, w)$ is given by $-2 s \in Z$ 。

\section{HOLOMORPHIC BUNDLES OVER $S^{2} \times S^{2}$}

In dealing with complex space-times, one often encounters "complexified" functions of definite spin and conformal weight. Examples include the asymptotic shear of a complex null hypersurface and the related "cut functions" of Newman and co-workers. ${ }^{1,4}$

Intuitively, one simply proceeds by analytic continua- tion: If $f\left(\xi^{A}, \xi^{A^{\prime}}\right)$ is real analytic, and homogeneous of degree $(w-s, w+s)$ in $\left(\xi^{A}, \xi^{A^{*}}\right)$, then $f\left(\xi^{A}, \eta^{A^{*}}\right)$ is a holomorphic function of four complex variables, defined in an open neighborhood of the "real slice" $\eta^{A^{\prime}}=\bar{\xi}^{A^{\prime}}$ ). By analogy with the real case, one expects these functions to correspond to sections of certain holomorphic line bundles over the "complexification" of $S^{2}$. We shall see below that this implies a restriction on the possible values of $s$ and $w$.

To proceed rigorously, we consider the principal $C^{*} \times C^{*}$ bundle $\pi:\left(C^{2}-\{0\}\right) \times\left(C^{2}-\{0\}\right) \rightarrow P_{1}(C) \times P_{1}(C)$ $\cong S^{2} \times S^{2}$; the mapping $\pi$ sends the pair $\left(\xi^{A}, \eta^{A^{*}}\right)$ to $\left(\left[\xi^{A}\right],\left[\eta^{A^{\prime}}\right]\right)$. The base space should be regarded as the projective space of complex null directions at some fixed point in a complex space-time. We denote the total space of the bundle by $E$.

Notice that the points $\left(\left[\xi^{A}\right],\left[\eta^{A^{\prime}}\right]\right)$ of $P_{1}(C) \times P_{1}(C)$ are in one-to-one correspondence with the proportionality classes of nonzero, singular $2 \times 2$ matrices $\left[\xi^{A} \eta^{A^{\prime}}\right]$ 。 We shall use this identification in the following.

The only holomorphic representations of $C^{*} \times C^{*}$ on $C$ are given by $(\lambda, \mu) \rightarrow \lambda^{m} \mu^{n}$, where $m$ and $n$ are integers. We set $s=(m-n) / 2, w=-(m+n) / 2$; note that $w$ and $s$ are either both integer or half-integer depending on whether $m$ and $n$ have the same or opposite parity. Sections of the resulting bundles $\widetilde{B}(s, w)$ are in one-to-one correspondence with holomorphic functions on $E$ satisfying

$$
f\left(\lambda \xi^{A}, \mu \eta^{A^{2}}\right)=\lambda^{w-s} \mu^{w+s} f\left(\xi^{A}, \eta^{A^{\prime}}\right) .
$$

By restricting $\widetilde{B}\left(s, w^{\prime}\right) \rightarrow P_{1}(C) \times P_{1}(C)$ to each of the factors of the base, one easily shows that the Chern class of this bundle is given by $(x-s, w+s)$ $\in H^{2}\left(P_{1} \times P_{1}, Z\right) \cong Z \times Z$. Thus, if $\left(s, x^{\prime}\right) \neq\left(s^{\prime}, x^{\prime}\right)$, the bundles $\widetilde{B}(s, w)$ and $\widetilde{B}\left(s^{\prime}, w^{\prime}\right)$ are topologically, and hence analytically, inequivalent, a significant difference from the situation in the real case. Note, in particular, that none of these bundles is a product except for $\widetilde{B}(0,0)$.

\section{Example: The complex null cone}

In a real space-time, the nonzero null vectors at any given point form a trivial $R^{*}$-bundle over the 2 -sphere of real null directions; in a complex space-time, however, the analogous bundle is nontrivial. To see this, choose and fix a spin frame at the point in question, so that any nonzero null vector may be represented in the form $\xi^{A} \eta^{A^{\prime}} \neq 0$. Of course, the same vector may be written as $\pi^{A} \tau^{A^{\prime}}$, where $\pi^{A}=\alpha \xi^{A}$ and $\tau^{A^{\prime}}=\alpha^{-1} \eta^{A^{\prime}}$, for any $\alpha \in C^{*}$. The mapping $\xi^{A} \eta^{A^{\prime}} \rightarrow\left[\xi^{A} \eta^{A^{\prime}}\right]$ exhibits the complex null cone $C N$ as a $C^{*}$-bundle over $P_{1}(C) \times P_{1}(C)$.

Now consider the $C^{*}$-bundle obtained from $\widetilde{B}(0,1)$ by deleting the zero section. Recall that an element of this bundle is an equivalence class $\left\{\left(\xi^{A}, \eta^{A^{*}}\right), z\right\}$, with $z \boxminus C^{*}$ and $\left\{\left(\xi^{A}, \eta^{A^{\prime}}\right), z\right\} \sim\left\{\left(\lambda \xi^{A}, \mu \eta^{A^{\prime}}\right), \lambda \mu z\right\}$ for $(\lambda, \mu)$ in $C^{*} \times C^{*}$. It is clear that the mapping $\left\{\left(\xi^{A}, \eta^{A^{*}}\right), z\right\}$ $\rightarrow z^{-1} \xi^{A} \eta^{A^{\prime}}$ is a holomorphic bundle isomorphism of $\widetilde{B}(0,1)-\{$ zero section $\}$ onto $C N$. Thus the complex null cone is a nontrivial $C^{*}$-bundle over its space of generators. This means in particular that, in contrast to 
the real case, the complex null cone has no global cross-sections or "cuts." 12 This situation may be remedied by adding back the zero section in an appropriate way, as we shall see below.

\section{THE STRUCTURE OF COMPLEX NULL INFINITY}

The conformal compactification of complex Minkowski space is obtained as follows (see Ref. 13 for the analogous construction in the real case):

Define a complex metric on $C^{6}$ by $h(y, y)=\left(y^{0}\right)^{2}$ $-\left(y^{1}\right)^{2}-\cdots-\left(y^{4}\right)^{2}+\left(y^{5}\right)^{2}$, and let $N$ be the set of all nonzero null vectors. If $y \in N$, so is $\lambda y$ for all $\lambda \in C^{*}$, and the conformal compactification $C M=N / C^{*}$ is a well-defined compact complex 4-manifold in $P_{5}(C)$. The space $C M$ is well known to mathematicians as the Grassmann manifold of lines in $P_{3}(C)$. Denote the image of $y$ in $C M$ by its homogeneous coordinates $\left[y^{a}\right]$.

Complex Minkowski space, $C M^{4}$, is naturally embedded in $C M$, via

$$
\phi\left(z^{\mu}\right)=\left[z^{\mu}, \frac{1}{2}(1+z \cdot z), \frac{1}{2}(1-z \cdot z)\right], \quad z^{\circ} z=z^{\nu} z_{\nu} .
$$

It is easily checked that the image of $\phi$ contains all points of $C M$ except those for which $y^{4}+y^{5}=0$. These exceptional points have the form $\left[y^{\mu}, y^{4},-y^{4}\right]$, where $y^{\mu} y_{\mu}=0$ and not all $y^{a}=0$. The complex 3 -manifold obtained from them by deleting the singular point $I=[0,+1,-1]$ is called complex null infinity and denoted by $C$ l. Precisely as in the real case any null geodesic $\left\{z^{\mu}+\lambda b^{\mu}: \lambda \in C, b \cdot b=0\right\}$ in $C M^{4}$ has a unique "end point" on $C$ ? given by

$$
\lim _{\lambda \rightarrow \infty}\left[\phi\left(z^{\mu}+\lambda b^{\mu}\right)\right]=\left[b^{\mu}, z \cdot b,-z \cdot b\right] .
$$

Changing to spinor coordinates and putting $\zeta=y^{4}$ $=-y^{5}, C \&$ may be represented by the submanifold of $P_{4}(C)$ given by

$$
C \varrho=\left\{\left[\xi^{A} \eta^{A^{\prime}}, \zeta\right]: \xi^{A} \eta^{A^{\prime}} \neq 0, \zeta \in C\right\}
$$

which exhibits $C \&$ as a line bundle over $P_{1}(C) \times P_{1}(C)$. The projection is given simply by $\pi:\left[\xi^{A} \eta^{A^{\prime}}, \zeta\right]-\left[\xi^{A} \eta^{A^{\prime}}\right]$ and the vector space structure is defined as follows: If $\pi\left(\left[\xi^{A} \eta^{A^{\prime}}, z\right]\right)=\pi\left(\left[\sigma^{A} \tau^{A^{\prime}}, w\right]\right)$, there is a unique $\lambda \in C^{*}$ such that $\lambda \sigma^{A} \tau^{A^{\prime}}=\xi^{A} \eta^{A^{\prime}}$; we set

$$
\left[\sigma^{A} \tau^{A^{\prime}}, w\right]+\left[\xi^{A} \eta^{A^{\prime}}, \zeta\right]:=\left[\xi^{A} \eta^{A^{\prime}}, \lambda w+\zeta\right]
$$

We may now observe that the mapping $\left\{\left(\xi^{A}, \eta^{A^{A}}\right), \zeta\right\}$ $\rightarrow\left[\xi^{A} \eta^{A^{\prime}}, \zeta\right]$ defines a vector bundle isomorphism between $\widetilde{B}(0,1)$ and $C$. Thus, the difference between $C N$ and $C l$ is just the difference between a $C^{*}$-bundle and the naturally associated line bundle.

Using (4.2), we see that the zero section of $C Y$ is just the set of "end points" of all null geodesics passing through the point 0 in $C M^{4}$. From the standpoint of $I$, of course, it is the null cone at 0 which is "at infinity", so that $C \&$ minus its zero section is just the (ordinary) complex null cone of the point $I$.
In contrast to $C N, C 9$ admits a four-dimensional vector space of global sections; these are precisely the "good cuts." If $z^{A A}$ is an arbitrary point of $C M^{4}$, and $z^{A A^{\prime}}$ is an arbitrary point of $C M^{4}$, and $z^{A A^{\prime}}+\lambda \xi^{A} \eta^{A^{\prime}}$ is a null geodesic through $z^{A A^{\prime}}$, it intersects $C Y$ in the point $\left[\xi^{A} \eta^{A^{\prime}}, z_{A A^{\prime}} \xi^{A} \eta^{A^{\prime}}\right]$ [see (4.2)]. Notice that this point depends only on the direction $\left(\left[\xi^{A}\right],\left[\eta^{A^{\prime}}\right]\right)$ of the geodesic through $z^{A A^{\prime}}$. As the direction varies, we obtain the global cross section

$$
Z:\left[\xi^{A} \eta^{A^{\prime}}\right]-\left[\xi^{A} \eta^{A^{\prime}}, z_{A A^{\prime}} \xi^{A} \eta^{A^{\prime}}\right]
$$

of $C$. The section $Z$ can be concisely represented as a holomorphic function on $E$ :

$$
Z\left(\xi^{A}, \eta^{A^{*}}\right)=z_{A A^{\prime}} \xi^{A} \eta^{A^{\prime}}
$$

Conversely, any section of $C$ defines a holomorphic function homogeneous of degree $(1,1)$ on $E=C^{2}-\{0\}$; since any such function is automatically an entire function (Hartog's theorem, Ref. 14, pp. 50ff。), it must be a polynomial such as that in (4.6). Thus the sections of $C 9$ are parametrized by the points of $C M^{4}$, and form a four-dimensional vector space, as asserted. In any trivialization of $C l$, the local representatives $u_{\alpha}$ $=Z\left(\zeta_{\alpha}, \eta_{\alpha}\right)$ of $Z$ will be characterized as solutions to the differential equation

$$
\delta_{\alpha}^{2} Z=0 \text {. }
$$

We conclude with a few remarks concerning asymptotic twisters. At any point $z^{A A^{\prime}}$ of $C M^{4}$, the null cone is generated by totally null two-planes (twistor surfaces $^{4}$ ) of the form

$$
\left\{z^{A A^{\prime}}+\xi^{A} \eta_{0}^{A^{\prime}}: \eta_{0}^{A^{\prime}} \text { fixed } \neq 0, \xi^{A} \neq 0\right\} 。
$$

This surface intersects $C \oint$ in the set $\left\{\left[\xi^{A^{A}} \eta_{0}^{A^{*}}, z_{A A^{\prime}} \xi^{A} \eta_{0}^{A^{\prime}}\right]\right\}$, a projective asymptotic twistor of $C M^{4}{ }^{2}$ Clearly, each section of $C l$ is ruled by asymptotic twistors. Of course, $C l$ itself is also generated by twistor surfaces. These have the form (in the valence $\left[\begin{array}{l}1 \\ 0\end{array}\right]$ case) $\left\{\left[\xi^{A} \eta^{A^{\prime}}, z\right]: z \in C, \xi^{A}\right.$ fixed $\left.\neq 0, \eta^{A^{\prime}} \neq 0\right\}$, and are actually line bundles over $P_{1}(C)$. They correspond to the restriction of $C g \rightarrow P_{1}(C) \times P_{1}(C)$ to $\left\{\left[\xi^{A}\right]\right\} \times P_{1}(C)$. Similar remarks apply to the valence $\left[\begin{array}{l}0 \\ 1\end{array}\right]$ asymptotic twistors.

${ }^{1}$ E. T. Newman, Gen. Rel. Grav。 7, 107 (1976)。

${ }^{2} \mathrm{R}$. Penrose, article in Quantum Gravity, edited by C. Isham et al. (Oxford U. P., London, 1975).

${ }^{3}$ A. Held, E. T. Newman, and R. Posadas, J. Math. Phys. 11, 3145 (1970).

${ }^{4}$ R. Hansen and E. T. Newman, Gen。 Rel. Grav. 6, 361 (1975).

${ }^{5}$ D. Husemoller, Fiber Bundles (McGraw-Hill, New York, 1966).

iS. Kobayashi and K. Nomizu, Foundations of Differential Geometry, I, II (Wiley, New York, 1963, 1969).

$7\{p, z\}$ is a vector at $\pi(\phi) . z$ is its "component" in the "frame" $p$, while $\rho\left(g^{-1}\right) z$ is its component in the frame $p g$.

${ }^{8}$ E. T. Newman and R. Penrose, J. Math. Phys. 7, 863 (1966).

${ }^{9}$ See either Ref. 10 or Ref. 11. The space $C^{2}-\{0\}$ is the homogeneous space $\operatorname{SL}(2, C) / T^{2}$, where $T^{2}$ is the Abelian group 


$$
\left\{\left[\begin{array}{ll}
1 & z \\
0 & 1
\end{array}\right]\right\}: z \in C
$$

the sphere is the homogeneous space $\operatorname{SL}(2, C) / C^{*} \circ T^{2}$. If we induce with the representation $(s, w):(\lambda, z) \rightarrow \lambda^{s-w} \bar{\lambda}^{-(s+w)}$, the resulting $C$-valued functions on $\operatorname{SL}(2, C)$ are constant on the cosets of $T^{2} ; \mathrm{i}, e_{.}$, they are functions on $C^{2}-\{0\}$. This is the group-theoretical origin of the bundles $B(s, w)$.

10T. M. Gel'fand, et al., Generalized Functions, Vol. 5 (Academic, New York, 1966).
${ }^{11}$ N. Wallach, Harmonic Analysis on Homogeneous Spaces (Dekker, New York, 1973).

${ }^{12}$ A principal bundle is trivial $\Leftrightarrow$ it admits a global cross section. Any global cross section of $\widetilde{B}(0,1)-\{$ zero section $\}$ gives rise to a cross section of $\widetilde{B}(0,1)$ itself; but as we shall see in the next section, any cross section of $\widetilde{B}(0,1)$ is represented by a polynomial and therefore has zeroes. Thus the principle bundle has no global cross section.

${ }^{13} \mathrm{D}$. Lerner, J。 Math. Phys. 18, 1812 (1977)。

${ }^{14} \mathrm{R}$. Narasimhan, Several Complex Variables (University of Chicago Press, Chicago, 1971). 